\title{
THE LANGUAGE STATUS OF THREE CHILDREN AT THE SAME AGES
}

\author{
By W. G. BATEMAN, University of Montana
}

Although, in various publications, information in growing quantity has been published about the language development of the individual child, little appears to be available regarding groups of children. Usually each author observes only one subject who is, in most cases, an only child. This leads to the exclusion of certain factors such as individual differences, the influence of children upon one another, the reaction to different environments, etc. Holden (11) listed the words used by his two daughters at the same age; W. and C. Stern (19) have published an interesting volume on some phases of language development using their three children as subjects; Gale (8) has recorded the vocabularies with some comments on three children of the same family; Bateman (1-2-3) is noting the various steps in the acquisition of speech by his two children, but these seem to exhaust the references. This paper discusses the ability in talking gained by three children from birth to twenty-eight months, the subjects being the author's two daughters and niece.

Some of the data have already been published (I) but will be used here for comparison. In two other articles (2-3) will be found more extended information regarding two of the children.

\section{BIOGRAPHICAL.}

Jane and Anne were 28 months old on July 14, 1913, and March 28, 1915, respectively. Both are large, active and healthy, having a record of almost perfect health. At the above age by the Binet scale Jane ranked 3.5 and Anne 3 . Racially their inheritance is rather mixed in a composition of English, Welsh, Dutch and German, the first predominating. Both parents are college graduates and teachers. Up to the time these observations were taken no particular efforts were taken along the lines of language development. The parents, however, endeavored to speak distinctly and correctly, encouraged all attempts by the children in this direction and discouraged "baby-talk."

Daphne Ada Wyman, first cousin of the above, was born 
at Racine, Wisconsin, May 23, 1913. She is a large, sturdy child having had practically no ailments since birth. She has lived in California since 3 months old almost continuously with her parents although on a few occasions it was necessary for her to live with friends for a time. These absences from home loosened considerably discipline and training. Both her parents hold college diplomas. The one has been a teacher, the other is a Boys' Director in the Young Men's Christian Association. Her father is of German-English descent and the mother of English-Welsh. She received no particular training in speech; some points in this connection will be brought out later. At 28 months her responses to the Binet tests gave her a rank of 3 .

Thus the general inheritance and environment of the three children are the same. Physically they resemble each other, showing their relationship more or less plainly. In general mental ability at 28 months they were on a par except that each child had some traits not shown by the others in so marked a degree. For instance, Jane had considerable ability in observation and concentration and a marked ability to do things with her hands; Anne had better general muscular control with consequent ease and abandonment of movement as well as decided mental alertness; Daphne had developed a sense of order quite uncommon since she knew the proper place for almost everything in the home and spent much energy in keeping it there. Temperamentally, on the other hand, all three were very different. Jane displayed the more calm and even disposition; Anne was more impulsive and at times tempestuous; Daphne more variable in mood and more emotional. All were (and are) strong-willed but while Jane was most cheerful under discipline and sought to gain her ends by more gentle methods, Anne did not accept discipline gracefully using persistence as her main reliance, and Daphne was more the rebel and fighter combating, ofttimes strenuously, what did not conform to her wishes. These differences in certain abilities and traits will be seen to have had an influence upon the language development in each case.

DATA.

The author's children were continuously under his observation and the cousin as well during five weeks of the twentyseventh and twenty-eighth months. Other data concerning the latter were furnished by her mother. The vocabularies were obtained by writing down a large part of each child's conversation during two weeks, every effort being made to have the list complete. None of the children was conscious of the close scrutiny. 


\section{STATUS AT TWELVE MONTHS.}

Time of First Word. All three children used the first word with definite meaning at about the same time-Jane at ten and one-half, Anne at ten and Daphne at eleven months. These words were 'hello,' ' daddy' and 'bye-bye,' respectively.

Babbling. This was practiced constantly by all three children, most so by Anne and least so by Daphne, but only Jane and her sister found enjoyment in stringing together long series of similar sounds such as 'uggle-guggle-wuggle.'

Vocabularies at Twelve Months.-Jane-10 words: Nouns-chow,* daddy, dog, pot, toast. Verbs-go, see. Adjectives-pretty, good. Interjection-hello.

Anne-9 words: Nouns_-ball, chow, daddy. Verb)—see. Adjectivedear. Adverbs - down, good-bye. Interjections-boo, hello.

Daphne-8 words: Nouns - chow, dada, dog, mama, pot. Pronominal-what's-that (always used together). Interjections-bye-bye, oh.

Comparison. The following table gives information about the vocabularies of one-year-old English speaking children.

\section{TABLE I}

\begin{tabular}{|c|c|c|c|c|c|c|c|}
\hline Authority & Ref. & $\begin{array}{l}\text { No. of } \\
\text { words }\end{array}$ & Nouns & Verbs & Adjs. & Advs. & Interjs. \\
\hline Hall. & 10 & & 19 & 2 & 0 & 0 & 3 \\
\hline Mickens. & .13 & 3 & 2 & 0 & 0 & 0 & 1 \\
\hline Moore & 14 & 6 & 6 & 0 & 0 & 0 & 0 \\
\hline Pelsma & .17 & 10 & 7 & 1 & 0 & 1 & 0 \\
\hline Tracy. & .20 & 10 & 6 & 0 & 2 & 2 & 0 \\
\hline 30 & 5 & ${ }_{7}^{8}$ & 7 & 0 & 0 & $\stackrel{1}{0}$ & 0 \\
\hline Gra & 9 & 3 & 2 & 0 & 0 & 1 & 0 \\
\hline
\end{tabular}

The average number of words is 9; if the highest and lowest are left out, the average of those remaining is 8 . The three children, then, used not only about the same number of words but this close to the average.

The composition of the three lists, however, is quite different, as shown in the following table.

TABLE II

\begin{tabular}{|c|c|c|c|c|c|c|c|}
\hline Subject & $\begin{array}{l}\text { No. of } \\
\text { words } \\
10\end{array}$ & Nouns & $\begin{array}{l}\text { Verbs } \\
20 \%\end{array}$ & Adjs. & Advs. & $\begin{array}{l}\text { Interjs. } \\
10 \%\end{array}$ & Pronom. \\
\hline Ann & 0 & $33 \%$ & $11 \%$ & $11 \%$ & $23 \ddot{\%}$ & $23 \%$ & \\
\hline Daphne & 8 & $62.5 \%$ & & & & $25 \%$ & $12.5 \%$ \\
\hline
\end{tabular}

Thus Anne has the most varied vocabulary and Daphne the simplest. The pronominal expression, ' what's that,' used by the latter is quite rarely used by such young children, similar words not appearing in any of the above listed vocabularies.

* Chinese word used by the children to mean something to eat. 
The lack of verbs in Daphne's list is in agreement with those of most other children and the use of adjectives by Jane and Anne rather unusual.

Enunciation. In using her ten words Jane spoke very clearly in a careful fashion and with an intonation quite unlike other babies. She found little trouble in imitating simple words which was rather an amusement.

Anne spoke with considerably less clearness, running the sounds together and especially broadening the vowel sounds. There was a decided tendency to clip the last sound from words, particularly the ' $\mathrm{g}$ ' in 'ing.' She soon tried to correct this voluntarily. She spent much time in chattering and imitating.

Daphne pronounced three of her eight words correctlybye-bye, mama, dada. Chow was ' dow,' oh became ' lo,' pot and dog lost the final sound and the expression, what's that, was 'washa.' Some of these will be noted again. While babbling a good deal she did not imitate as much as other children.

\section{DEVELOPMENT FROM 12 TO 28 MONTHS.}

Daphne. At twelve months Daphne was chattering in a lively fashion and using 8 words with definite and proper meaning. During the next six weeks this number was much increased but during the rest of the fourteenth month and all of the fifteenth fewer words were in use, not more than 20. She seemed to be doing all she could to simplify the language. Anything to eat or drink was 'good' with a long ' $\mathrm{mm}$ ' after it. The phrase, 'what's that,' was never used, only ' that' accompanied by pointing for anything she wanted. The nouns themselves were seldom used. The foreign word ' chow' was lost in neglect as well. During this time she was more or less strenuously engaged in learning to walk. The whole period is a good example of the so-called "plateau " for after walking was accomplished there was a decided expansion in the language development. The most significant loss during the recovery was the failure of the desire to imitate. Whereas before the plateau stage she would repeat things she heard, this was rarely true afterwards. Little attention was paid to conversation around her, few pains were taken on her part to speak clearly and from a rather overuse of vocalistics she became, on the contrary, decidedly silent. These changes had a marked influence on her later status. The materials gained for self-expression, however, increased largely during this period.

The sentence form did not appear until the seventeenth 
month when the simplest forms were first used. They have remained undeveloped due to the late or non-acquisition of some of the parts of speech. After the second year compound sentences were in use but a slight pause took the place of conjunctions. Six months elapsed between the use of the first word and the first sentence.

Anne. As shown above, Anne began her second year with a stock of nine words and as time went on rapidly acquired new terms. She imitated much that she heard about her and practiced certain words with much energy, seeming to realize that her methods of pronunciation were not quite the same as those of grown-ups.

Simple sentences were first used in the fifteenth month. By the twenty-fourth month all the parts of speech were in use, the conjunction coming only at the date mentioned. Before this a slight pause or repetition took the place of ' and.' At this time, then, the child began to make all types of sentences. Five months passed between the first word and first sentence.

Beginning in the nineteenth month and lasting about two months there was a marked change in the child's attitude toward talking. She no longer cared to imitate either spontaneously or by coaxing. Very few new words were learned and the greatest economy was practiced in sentence structure. The whole affair seemed a decided retrogression. However, Anne was as active and alert as ever in other directions. Physically, she was much engaged with dentition which was somewhat delayed. As soon as the molar teeth had erupted she suddenly began to chatter again, making very rapid progress, so that all the lost ground was easily recovered. New words were used literally by dozens. This rapid development after the so-called plateau stage has been noted by a number of others.

At two years Anne was using 407 words and had a maximum vocabulary of 497 which compared favorably with others published for children of this age (3). During the next four months she increased the number somewhat over 50 per cent., gained in ability to use the sentence form and improved markedly in enunciation.

Jane. After once beginning to talk Jane made consistent progress, not interrupting her course as did the other two. At fifteen months she was using 75 words, at eighteen months one hundred more and at two years about 300 were in use. Among the parts of speech, however, the conjunction was missing so that the more complex sentence forms were not possible. Following the practice of the others, Jane used slight pauses and repetition to take the place of connectives. 
The sentence in its simplest form first came largely into use in the sixteenth month, and during the next six months was developed as far as the child's limited materials allowed. Five months intervened between the first word and first sentence.

But although Jane showed no well marked plateau stage two variations in method should be noted. During the three months from the fifteenth to the eighteenth she discontinued active imitation and declined to use two Chinese words which heretofore had been of as much service as others. However, she went right on talking and used new words which she learned by direct observation without practice. After the eighteenth month, however, when she had suddenly learned to walk, she began both imitation and the use of the tabooed foreign words. At the beginning of the twenty-fourth month the second phase of individual speech activity began. The child now took to speaking long strings of sounds well modulated and phrased but quite unintelligible although accompanied by many expressive inflections and turns of the voice. It soon appeared that she was anxious to really converse and to hold up her end of the conversation and indeed at a distance it sounded very well. She persisted in this imitation until by dint of acquiring many new words and ideas she was really able to make it serve her, which result was reached in about two months. Since that time she has been for a little child unusually direct and concise in her talk so that a few have termed her " an old-fashioned girl."

Comparison. In general during this interval Anne made the most rapid progress and Daphne somewhat the slowest. Some reasons for this will be discussed later.

While Jane did not pass through any well-defined plateau stage she did display more interest in language improvement after learning to walk. But Anne, like Daphne, went through a period of marked deterioration in speech activity but connected apparently with dentition rather than walking. Some authorities have thought such neglect of talking to be caused by diversion of energy to other channels.

Jane first used simple sentences in the sixteenth month, Anne in the fifteenth and Daphne in the seventeenth. From this beginning to complete and complex sentences took fourteen months for Jane, ten for Anne and was far from complete after ten to eleven in Daphne's case.

It is instructive to note the long time during which only single words were used, 5 months for the two sisters and 6 for the cousin. Stern considers this to show how well the single word sentence can suffice for the child's needs and that it does not emphasize the slowness of development. Some 
children retain such simple usage for much longer periods, even 11 to 12 months (Drever, Major) in some cases.

The greatest difference among the children is seen to be in the spontaneous interest in speech activity shown respectively by the three youngsters.

\section{STATUS AT 28 MONTHS.}

The vocabularies were compiled according to the following rules: 1, no proper nouns; 2 , no plurals unless the singulars were not used; 3 , all forms of pronouns are included; 4, no variants of verbs or adjectives unless sometimes from different roots; 5 , the same word may be listed more than once according to its grammatical use by the child; 6, only words used with at least some approximation to correct meaning are listed; 7, words deviating from usage are in quotation marks followed by an explanatory word in brackets; 8, words used in common by all three children are in italics.

\section{JANE'S VOCABULARY AT 28 MONTHS}

A. Nouns-apple, apron, arm.

Verb-am.

Adjectives-a, all, awake.

Adverbs-alright, around, away.

Preposition-about.

Interjection-ah.

B. Nouns-back, bacon, bag, ball, basket, bath, bathroom, beans, bed, beet, bib, bird, biscuit, bite, blanket, block, book, bone, bottle, bouquet, box, bread, broom, brush, bucket, buckle, button.

Verbs-bite, break, brush, build, button.

Adjectives-beautiful, big, broken.

Adverbs-back, better, backward.

Interjection-boo.

C. Nouns-cabinet, cake, can, candy, car, carrots, cart, cascara, cat, cellar, chair, chain, charcoal, cheese, cherries, chick, chocolate, chow, clock, closet, clothes, clothes-pin, coal, coal-shed, coat, cocoa, coffee, comb, cotton, cork, couch, crayon, cream, "crax" (crackers), cube, cup.

Verbs-can, can't, carry, clean, close, come, cook, cough, crawl, cry, cut.

Adjectives-chocolate, clean, cold, crooked.

D. Nouns-daddy, diaper, dinner, ditch, dog, doll, door, dress, drink, duck

Verbs-dance, do, don't, draw, dress, drink, drop.

Adjectives-dear, dirty.

Adverb-down.

Interjection-darn.

E. Nouns-ear, egg, enough, eye.

Verbs-eat, excuse.

F. Nouns-face, feather, feet, fire, fish, floor, flower, fly, fork.

Verbs-fall, feel, fit, fix.

Adjectives-foolish, funny. 
G. Nouns-garter, girl, glass, go-cart, "gran" (grandma), grass.

Verbs-get, go, gulp, gurgle.

Adjective-good.

Adverbs-good-bye, good-night.

H. Nouns-hair, hand, handle, "hankie" (handkerchief), hat, head, heel, hole, home, honey, house, horse.

Verbs-hang, have, hear, help, hit, hug, hurry, hurl.

Adjectives-heavy, high, hot, hurt.

Adverb-here.

Pronoun-him.

Interjection-hello.

I. Verb-is.

Pronouns-I, it.

Preposition-in.

J. Nouns-jacket, jar, jelly.

Verb-jump.

Adverb-just.

K. Nouns-key, kiss, kitchen, knee.

Verbs-keep, kick, kiss, knock.

L. Nouns-lawn, leg, lid, light, "lots".

Verbs-lay, laugh, lean, let, lie (recline), like, lock, look.

Adjectives-long, lost, lovely.

Adverb-lot.

M. Nouns-macaroni, man, meat, milk, mother, moon, mosquito, mouth, mouthful, mush.

Verbs-move, must.

Adverb-more.

Pronouns-me, my.

N. Nouns-"nakum" (napkin), neck, needle, nightshirt, nose.

Adjectives-nasty, naughty, new, nice.

Adverbs-no, not, now.

0 . Verb-open.

Adjectives-old, one, other.

Adverbs-off, on, out, over.

Prepositions-of, on.

Interjections-oh, ouch.

P. Nouns-pail, pair, pan, paper, paste, pease, pencil, pie, piece, pillow, pin, plate, plenty, plug, pocket, pot, prunes, pudding, pup.

Verbs-pat, pick, play, put.

Adjectives-pink, precious, pretty.

Interjection-"plunk" (kerplunk).

R. Nouns-rabbit, rake, rattle, ribbon, rice, rockingchair, rompers, room, rubber.

Verbs-rain, rake, reach, rock, run.

Adjective-ready.

Adverb-right.

S. Nouns-salt, salve, sandal, school-side, shirt, shoe, shovel, slipper, soap, socks, sound, soup, spoon, stair, step, sticker, stove, strawberries, string, suei, chow, sugar.

Verbs-say, see, sew, sit, shall, shut, sleep, smell, spank, spill, splash, stand, step, sweep.

Adjectives-silly, soaking.

Adverbs-so, some.

Pronoun-she. 
T. Nouns-table, table-cloth, taste, tea, teeth, things, tie, toast, tongue, town, train, tray, tree, trunk, tub.

Verbs-take, talk, tear, thank, tickle, tip, try, turn.

Adjectives - that, the, thin, this, thirsty, tin, tired, two.

Adverbs-there, through, too.

Pronouns-that, this.

Preposition-to.

Interjection-there.

U. Noun-umbrella.

Adverb-up.

Pronoun-us.

V. Nouns-vase, vaseline.

W. Nouns-walk, washrag, water, wood.

Verbs-wake, walk, want, wash, would.

Adjectives-warmer, wet, white, wrong.

Adverbs-"wayup," what, where.

Pronoun-we.

Interjections-whoa, whoop.

Y. Adverts-yes.

Pronouns-you, your.

The following table shows the number of words of each initial letter classified as to grammatical form, the total number of words beginning with each initial letter, and, finally, the total number of words in the above list:

TABLE III

Initial Nouns Verbs Adjs. Advs. Prons. Preps. Interjs. Tota

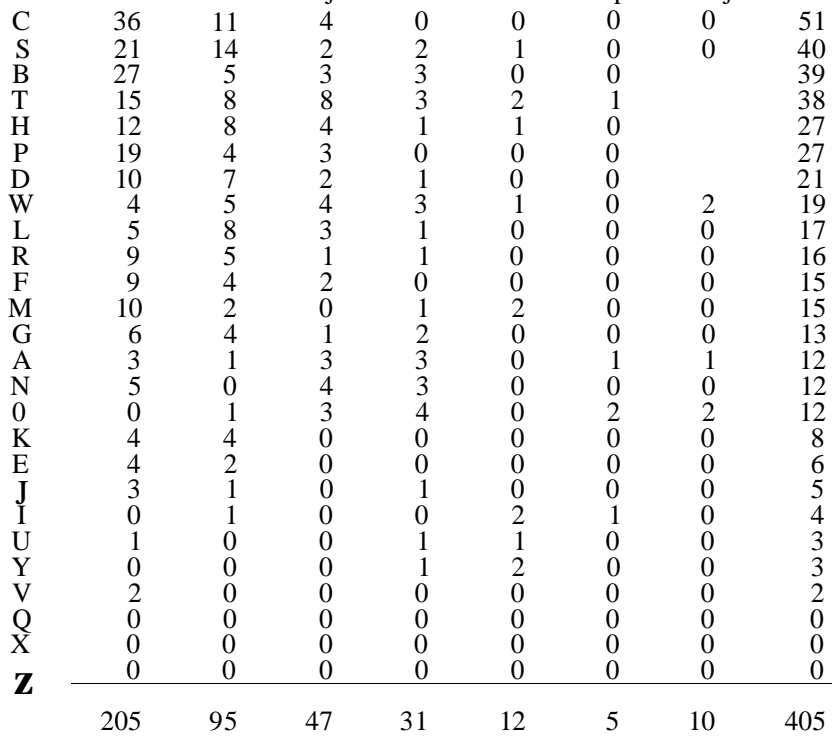


Table IV shows the percentage each part of speech is of the total number of words.

\section{TABLE IV}

Nouns Verbs Adjs. Advs. Prons. Preps. Interjs. Total

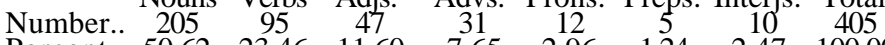

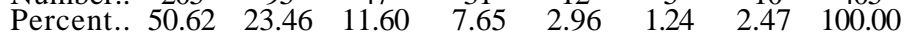

The next table shows the number of words beginning with each initial sound (as contrasted in some cases with initial letter).

\section{TABLE V}

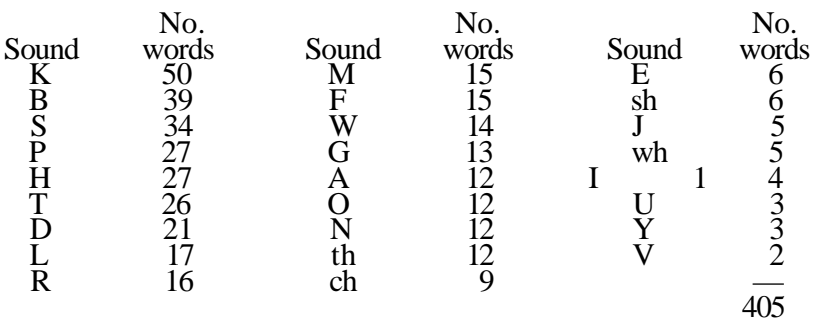

Proper Nouns.-Billie, Grand-ranch, Henry, Jane, Patsy, Percy, Puck, Roger, Rose, Sam, Squire.

If these were included in the vocabulary the total number of words would be 405 plus 11, or 416, of which the proper nouns would constitute 2.64 per cent.

Total number of words of all kinds:

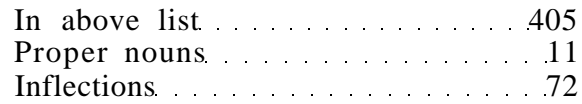

\section{8}

\section{ANNE'S VOCABULARY AT 28 MONTHS}

A. Nouns-accident, apple, apron, "aminal" (animal), anything, arm, ashes, automobile.

Verbs-am, ask.

Adjectives-a, all, awake, asleep.

Adverbs-again, alright, away.

Conjunction-and.

B. Nouns-baby, back, bacon, ball, banana, basket, bath, bathroom, bead, beans, bear, bed, bell, belt, bench, bib, bird, birthday, biscuit, block, bogie, bone, book, bottle, box, boy, bread, broom, brush, buckle, bug, bundle, butter, button.

Verbs-bang, back, bark, bite, bounce, break, bring, brush, buckle, build, bump, burn, button.

Adjectives-bad, big, black, blue, broken.

Interjections-bang, boo. 
C. Nouns-cake, can, candle, candy, cap, car, carrot, cart, casserole, cat, ceiling, cellar, chair, chain, cheek, cherry, cheese, chicken, chin, chow, clock, closet, clothes, coal, coalbucket, coat, cocoa, coffee, coffeepot, comb, comfort, cookie, cork, corner, cotton, couch, cow, cracker, crumb, cup.

Verbs-can, can't, call, carry, catch, chew, choke, clean, climb, come, cook, cough, cry, cut.

Adjectives-clean, cold, cute.

Adverbs-careful, close.

D. Nouns-daddy, desk, dimple, diningroom, dinner, dirt, dish, ditch, dog, doll, dollie, door, doughnut, drawer, dress, dressing gown, drink, duck, duster.

Verbs-dance, do, don't, dress, drink, drop, dry.

Adjectives-dirty, dry, dizzy.

Adverb-down.

E. Nouns-ear, egg, elbow, elephant, enough, eye.

Verbs-eat, excuse.

F. Nouns-face, fat, feather, finger, fingernail, fire, fish, floor, flower, flour, flute, fly, foot, fork, frog, fun, furnace.

Verbs - fall, fasten, feel, fill, find, fix.

Adjectives-fine, full, funny.

Adverb-fast.

Preposition-for.

G. Nouns-garter, girl, glass, glove, goat, gocart, goop, grass, gravy.

Verbs-get, give, go, grunt, gulp.

Adjective-good.

Adverbs-good-bye, good-night.

Interjection-Gesundheit.

H. Nouns-hair, hand, handle, "hankie" (handkerchief), hat, head, hole, home, honey, hook, horn, horse, house.

hurt.

Verbs-have, hang, hear, help, hiccough, hide, hold, hug, hurry,

Adjectives-heavy, high, hot, hungry.

Adverbs-hard, here.

Pronouns-he, her, herself, him, himself, his.

Interjection-hello.

I. Nouns-ice-cream, iron.

Verbs-is, isn't, iron.

Adverbs-in, indoors.

Pronouns-I, it.

Preposition-in.

J. Nouns_-jar, jelly, joke, juice, junket.

Verb-jump.

Adverb-just.

K. Nouns-key, kettle, kiddie, kitchen, kiss, knee, knife.

Verbs-keep, kick, kiss, knock.

Adverb-kindof.

L. Nouns-lap, leg, leggins, lemon, letter, light, load.

Verbs-laugh, let, lick, lie (recline), like, listen, look, love.

Adjectives-last, little, long, lost.

M. Nouns-macaroni, man, mat, meat, milk, milkman, minute, moon, more, mother, mouth, mouse, mud, muff.

Verbs-make, meet, move, must.

Adverb-more.

Pronouns-me, mine, my. 
N. Nouns-"nakum" (napkin), nail, neck, necklace, needle, night, nightgown, noise, noodles, nose, nut.

Verb-need.

Adjectives-nasty, naughty, new, nice.

Adverbs-nicely, next, no, not.

O. Noun-orange.

Verb-open.

Adjectives—old, one, orange, other.

Adverbs-off, on, out, outdoors.

Pronoun-one.

Prepositions-of, on, out, over.

Interjection-oh.

P. Nouns-pail, paint, pan, "pansweep" (dustpan), paper, part, party, path, pease, peach, pencil, pendant, penny, petticoat, piano, picnic, picture, pie, piece, pig, pillow, pin, place, plate, plug, pocket, pocket-book, poker, pomelo, porch, pot, potato, pudding

Verbs-parade, pat, pass, peel, pick, pinch, play, point, pour, pull, push, put.

Adjectives-pink, poor, pretty.

Adverbs-please, pretty.

Interjection-peek-a-boo.

Q. Adverb-quick.

R. Nouns-rabbit, rag, raisin, reins, ribbon, rice, ride, rocking-chair, rompers, room, rope, rubber, rug.

Verbs-rain, reach, read, remember, ride, rock, rub, run.

Adjectives-ready, real, right.

S. Nouns-salt, salve, sand, sandal, sandpile, sandwich, sauce, scalawag, schoolside, scissors, scratch, screwdriver, see-saw, shade, share, sheep, sheet, shirt, shoe, shoofly, shop, shovel, side, sister, skin, skum, slipper, sled, smack, smoke, snap, snow, soap, some, something, song, soup, spider, splinter, spoon, spring, step, stick, stocking, stone, story, stove, string, suei'chow, sugar, sun, sweater, swing.

Verbs-say, scratch, see, separate, serve, sew, shave, shine, shut, sing, sit, slap, sleep, smell, sneeze, spank, spit, splash, squeak, squeeze, stand, step, stop, sweep, swing.

Adjectives-sticky, still, sweet.

Adverbs-so, some, sometimes, still.

Pronouns-she, some.

T. Nouns-table, table cloth, tail, tea, tea party, teapot, teeth, thimble, things, thread, thumb, tick, tie, time, tit-bit, toast, tow, tongue, top, towel, tray, tree, trunk, tub, turkey, turn.

Verbs-take, talk, taste, tear, tell, thank, throw, tickle, tie, touch, try, turn.

Adjectives - that, the, these, thirsty, this, tired, two.

Adverbs-there, through, tonight, too.

Pronouns-that, this, these, they.

Preposition-to.

Interjection-there.

U. Nouns-"umerbella" (umbrella), underwear.

Verbs-undress, use.

Adverb-up.

Pronoun-us.

Preposition-under.

V. Noun-vaseline. 
W. Nouns-wagon, wall, washrag, water, way, wheel, wheelbarrow, whiskers, wind, window, wire, wood.

Verbs-wait, wake, walk, want, wash, wheel, wiggle, will, wipe, won't, work, write.

Adjectives - warm, well, wet, white.

Adverbs - way, when, where, why.

Pronouns-we, what (inter), who.

Preposition-with.

Interjection-why.

Y. Nouns-yarn, yellowbell.

Adverbs-yes, yesterday.

Pronouns-you, yours, yourself.

Interjection-yes sir.

Table VI shows the number of words of each initial letter classified as to grammatical form, the total number of words beginning with each initial letter, the total of each part of speech, and finally the total number of words in the above list:

\section{TABLE VI}

InitialNouns Verbs Adjs. Advs. Prons. Preps. Conjs. Interjs. Total

\begin{tabular}{lrrrrrrrrr}
$\mathrm{S}$ & 53 & 25 & 3 & 4 & 2 & 0 & 0 & 0 & 87 \\
$\mathbf{C}$ & 40 & 14 & 3 & 2 & 0 & 0 & 0 & 0 & 59 \\
$\mathrm{~B}$ & 26 & 12 & 7 & 4 & 4 & 1 & 0 & 1 & 55 \\
$\mathrm{P}$ & 34 & 13 & 5 & 0 & 0 & 0 & 0 & 2 & 54 \\
$\mathrm{~W}$ & 33 & 12 & 3 & 2 & 0 & $\bullet$ & 0 & 1 & 51 \\
$\mathrm{H}$ & 13 & 12 & 4 & 4 & 3 & 1 & 0 & 1 & 37 \\
$\mathrm{D}$ & 19 & 7 & 4 & 2 & 6 & 0 & 0 & 1 & 36 \\
$\mathrm{~F}$ & 17 & 6 & 3 & 1 & 0 & 0 & 0 & 0 & 30 \\
$\mathrm{R}$ & 13 & 8 & 3 & 1 & 0 & 1 & 0 & 0 & 28 \\
$\mathrm{M}$ & 14 & 4 & 0 & 1 & 0 & 0 & 0 & 0 & 24 \\
$\mathrm{~N}$ & 11 & 1 & 4 & 4 & 0 & 0 & 0 & 0 & 22 \\
$\mathrm{~L}$ & 7 & 8 & 4 & 0 & 0 & 0 & 0 & 0 & 20 \\
$\mathrm{~A}$ & 8 & 2 & 4 & 3 & 0 & 0 & 1 & 0 & 19 \\
$\mathrm{G}$ & 9 & 5 & 1 & 2 & 0 & 0 & 0 & 1 & 18 \\
$\mathrm{O}$ & 1 & 1 & 4 & 4 & 1 & 4 & 0 & 1 & 16 \\
$\mathrm{~K}$ & 7 & 4 & 0 & 1 & 0 & 0 & 0 & 0 & 12 \\
$\mathrm{I}$ & 2 & 3 & 0 & 2 & 2 & 1 & 0 & 0 & 10 \\
$\mathrm{E}$ & 6 & 2 & 0 & 0 & 0 & 0 & 0 & 0 & 8 \\
$\mathrm{Y}$ & 2 & 0 & 0 & 2 & 3 & 0 & 0 & 1 & 8 \\
$\mathrm{~J}$ & 5 & 1 & 0 & 1 & 0 & 0 & 0 & 0 & 7 \\
$\mathrm{U}$ & 2 & 2 & 0 & 1 & 1 & 1 & 0 & 0 & 7 \\
$\mathrm{Q}$ & 0 & 0 & 0 & 1 & 0 & 0 & 0 & 0 & 1 \\
$\mathrm{~V}$ & 1 & 0 & 0 & 0 & 0 & 0 & 0 & 0 & 1 \\
\hline & 335 & 152 & 55 & 42 & 25 & 9 & 1 & 9 & 628
\end{tabular}

The next table gives the percentage each part of speech is of the total number of words.

TABLE VII

Nouns Verbs Adjs. Advs. Pms. Prps. Cnjs. Intjs. Total $\begin{array}{llllllllll}\text { Number.. } & 335 & 152 & 55 & 42 & 25 & 9 & 1 & 9 & 628\end{array}$

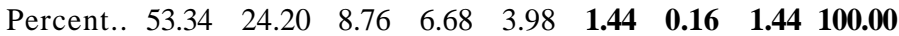


Table VIII shows the number of words beginning with each initial sound.

\section{TABLE VIII}

$\begin{array}{cccccc}\text { Sound } & \begin{array}{c}\text { No. } \\ \text { words }\end{array} & \begin{array}{c}\text { Sound } \\ \text { No. }\end{array} & \begin{array}{c}\text { Sound } \\ \text { words }\end{array} & \begin{array}{c}\text { No. } \\ \text { words }\end{array} \\ \text { S } & 70 & \text { N } & 23 & \text { I } & 10 \\ \text { K } & 56 & \text { M } & 22 & \text { E } & 8 \\ \text { B } & 54 & \text { L } & 19 & \text { Y } & 8 \\ \text { P } & 51 & \text { A } & 18 & \text { J } & 7 \\ \text { T } & 37 & \text { G } & 18 & \text { U } & 7 \\ \text { H } & 36 & \text { th } & 18 & \text { sc } & 6 \\ \text { D } & 30 & \text { O } & 14 & \text { Q } & 1 \\ \text { F } & 28 & \text { sh } & 13 & \text { V } & 1 \\ \text { W } & 28 & \text { wh } & 11 & & \text { 628 } \\ \text { R } & 24 & \text { ch } & 10 & & \end{array}$

Proper Nouns.-Billie, Catherine, Dickie, Doris, Fido, Grand-ranch, Gretchen, Isabel, Jack, Jane, Lewis, Logan, Louise, Lombard, Major, Marion, Percy, Rover, Salt Lake, Siebert, Smith, Underwood.

These 22 words if counted in the vocabulary would increase it to 650 , of which they would constitute 3.38 per cent.

Total number of words of all kinds:

In above list

Proper nouns.

\section{DAPHNE'S VOCABULARY AT 28 MONTHS}

A. Nouns-apple, arm, automobile.

Adjective-all.

Adverbs-again, almost, all, away.

Interjection-ah-ha.

B. Nouns-back, ball, bath, bathroom, bathtub, bead, beans, bed, bedroom, bee, beets, bib, bird, bite, block, book, bottle, bottom, box, boy, bread, brush, butter, button, buttonhook, bug.

Verbs-blow, brush, bump, button.

Adjectives-bad, big, blue, brandnew, broken.

Adverbs-better, big, bye-bye.

C. Nouns-cake, can, candy, canteloupe, car, card, cart, cat, chair, cheek, cheese, chick, clothes, clothespin, coat, cocoa, coffee, comb, corn, cow, cream, crumb, curl, curtain, cushion, custard, cup.

Verbs-can, catch, comb, come, cover, cry.

Adjective-clean.

D. Nouns—daddy, dirt, dog, dollie, door, drawer, dress, drink, duck.

Verbs-dance, dig, do.

Adjective-dirty.

Adverb-down.

E. Nouns-ear, egg, elbow, enough, eye.

Adjective-empty. 
F. Nouns-fig, finger, fingernail, fire, fish-man, flower, floor, fly, fork. Verbs-fall, feel, fix, fly.

G. Nouns-garter, girl.

Verbs-get, give, go.

Adjective-good.

H. Nouns-hair, hand, handle, "hankie" (handkerchief), hat, head, hole, horse, house.

Verbs-have, hurt.

Adjective-hot.

Adverb-here.

I. Nouns-ice-cream, ice-man.

Pronouns-I, it.

Preposition-in.

K. Nouns—key, kiss, kitchen, kitty, knee, knife, kodak.

Verb-kiss.

L. Nouns-lady, lady-bug, leg, letter, light, "lots".

Verbs-like, look, lose, love.

Adjective-little.

M. Nouns-macaroni, malted-milk, man, meat, milk, money, moon, more, mother, mouth, mud-pie, mush.

Verb-make.

Adverb-more.

Pronouns-me, my.

N. Nouns-neck, needle, night-gown, nose.

Adjective-new.

Adverbs-no, no-ma'am, not.

0 . Nouns-oilcloth, orange.

Verb-open.

Adjectives-old, one.

Adverbs-off, on, out, outdoors.

Pronoun-our.

Preposition-on.

Interjection-oh.

P. Nouns-pail, paper, pea, peach, pear, pencil, petticoat, piano, picture, piece, pillow, pin, pinecone, plate, plug, pocket, pocket-book, pot, potatoes, pudding.

Verbs-play, pour, push, put.

Adjectives-poor, pretty.

Adverb-please.

Q. Noun-quilt.

R. Nouns-rice, ring, rocking-chair, rompers, rug.

Verbs-rain, read, run.

S. Nouns-salt, sand, sandal, salve, sardine, scissors, shaving-brush, shirt, sheet, shoe, shovel, slipper, soap, sock, spool, spoon, stomach, strawberry, sugar, sugar-bowl, suit-case, sweater, swim, swimming-pool. spit, swim.

Verbs-say, see, sew, shave, shut, sing, sit, sleep, smell, spank,

Adjective-sore.

T. Nouns-table, tea, tea-pot, teddy-bear, teeth, thread, thumb, tie, toast, toe, tongue, tooth-brush, tooth-paste, towel, tree, tub.

Verbs-take, turn.

Adjectives-tiny, two.

Adverbs-there, through, too.

Pronoun-that. 
U. Nouns-umbrella, underwear.

Verb-unbutton.

Adverb-up.

W. Nouns-waist, watch, water, wood.

Verbs-walk, want, wash, wipe, write.

Adjective-wet.

Adverb-where.

Pronouns-we, what.

Y. Adverb-yes.

The following table shows the number of words of each initial letter classified as to grammatical form, the total number of words beginning with each initial letter, the total of each part of speech, and finally the total number of words in the above list.

TABLE IX

Initial Nouns Verbs Adjs. Advs. Prons. Preps. Interjs. Total

$\begin{array}{rrrrrrrrr}\text { B } & 26 & 4 & 5 & 3 & 0 & 0 & 0 & 38 \\ \text { S } & 24 & 12 & 1 & 0 & 0 & 0 & 0 & 37 \\ \text { C } & 27 & 6 & 1 & 0 & 0 & 0 & 0 & 34 \\ \text { P } & 20 & 4 & 2 & 1 & 0 & 0 & 0 & 27 \\ \text { T } & 16 & 2 & 2 & 3 & 1 & 0 & 0 & 24 \\ \text { M } & 12 & 1 & 0 & 1 & 2 & 0 & 0 & 16 \\ \text { D } & 9 & 3 & 1 & 1 & 0 & 0 & 0 & 14 \\ \text { F } & 9 & 4 & 0 & 0 & 0 & 0 & 0 & 13 \\ \text { H } & 9 & 2 & 1 & 1 & 0 & 0 & 0 & 13 \\ \text { W } & 4 & 5 & 1 & 1 & 2 & 0 & 0 & 13 \\ 0 & 2 & 1 & 2 & 4 & 1 & 1 & 1 & 12 \\ \text { L } & 6 & 4 & 1 & 0 & 0 & 0 & 0 & 11 \\ \text { A } & 3 & 0 & 1 & 4 & 0 & 0 & 1 & 9 \\ \text { K } & 7 & 1 & 0 & 0 & 0 & 0 & 0 & 8 \\ \text { N } & 4 & 0 & 1 & 3 & 0 & 0 & 0 & 8 \\ \text { R } & 5 & 3 & 0 & 0 & 0 & 0 & 0 & 8 \\ \text { E } & 5 & 0 & 1 & 0 & 0 & 0 & 0 & 6 \\ \text { G } & 2 & 3 & 1 & 0 & 0 & 0 & 0 & 6 \\ \text { I } & 2 & 0 & 0 & 0 & 2 & 1 & 0 & 5 \\ \text { U } & 2 & 1 & 0 & 1 & 0 & 0 & 0 & 4 \\ \mathrm{Q} & 1 & 0 & 0 & 0 & 0 & 0 & 0 & 1 \\ \mathrm{Y} & 0 & 0 & 0 & 1 & 0 & 0 & 0 & 1 \\ & 195 & 56 & 21 & 24 & 8 & 2 & 2 & 308\end{array}$

Table $\mathrm{X}$ gives the percentage each part of speech is of the total number of words.

TABLE $X$

Nouns Verbs Adjs. Advs. Prons. Preps. Interjs. Total

$\begin{array}{lllllllll}\text { Number.. } & 195 & 56 & 21 & 24 & 8 & 2 & 2 & 308\end{array}$

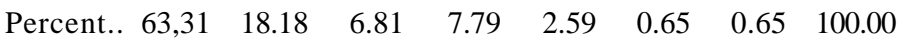

Table XI shows the number of words beginning with each initial. Owing to faulty enunciation this differs considerably from Table IX. 
TABLE XI

\begin{tabular}{|c|c|c|c|c|}
\hline Sound & $\begin{array}{l}\text { No. } \\
\text { words }\end{array}$ & Sound & $\begin{array}{c}\text { No. } \\
\text { words }\end{array}$ & Sound \\
\hline S & 53 & $\mathrm{D}$ & 19 & A \\
\hline B & 38 & M & 16 & $\mathrm{ch}$ \\
\hline $\mathrm{L}$ & 35 & W & 14 & $\mathrm{G}$ \\
\hline $\mathrm{K}$ & 31 & $\mathrm{~F}$ & 12 & sc \\
\hline $\mathrm{T}$ & 29 & $\mathrm{~N}$ & 11 & $\mathrm{U}$ \\
\hline $\mathrm{P}$ & 27 & 0 & 9 & \\
\hline
\end{tabular}

Proper Nouns.-Aunt Belle, Billie, Bill Veale, Daphne Ada, Dick-boy, Irene, Jane, Moore, Uncle Bill.

If these 9 words were included in the vocabulary the total would be increased to 317, of which these words would constitute 2.83 per cent.

In Table XII the figures for the three vocabularies are recapitulated for comparison.

TABLE XII

\begin{tabular}{|c|c|c|c|c|c|c|}
\hline & \multicolumn{2}{|c|}{ Jane } & \multicolumn{2}{|c|}{ Anne } & \multicolumn{2}{|c|}{ Daphne } \\
\hline & $\begin{array}{l}\text { Num- } \\
\text { ber } \\
205\end{array}$ & $\begin{array}{l}\text { Per } \\
\text { cent } \\
50.62\end{array}$ & $\begin{array}{c}\text { Num- } \\
\text { ber } \\
335\end{array}$ & $\begin{array}{l}\text { Per } \\
\text { cent } \\
53.34\end{array}$ & $\begin{array}{c}\text { Num- } \\
\text { ber } \\
195\end{array}$ & $\begin{array}{l}\text { Per } \\
\text { cent } \\
63.31\end{array}$ \\
\hline Verbs. & 95 & 23.46 & 152 & 24.20 & 56 & 18.18 \\
\hline Adjectives & 47 & 11.60 & 55 & 8.76 & 21 & 6.81 \\
\hline & 31 & 7.65 & 42 & $€ .68$ & 24 & 7.79 \\
\hline Pronouns & 12 & 2.96 & 25 & 3.98 & 8 & 2.59 \\
\hline Prepositions & 5 & 1.24 & 9 & 1.44 & 2 & 0.65 \\
\hline Conjunctions $\ldots$ & 0 & 0.00 & 1 & 0.16 & 0 & 0.00 \\
\hline Interjections... & 10 & 2.47 & 9 & 1.44 & 2 & 0.65 \\
\hline Total. & 405 & 100.00 & 628 & 100.00 & 308 & 100.00 \\
\hline
\end{tabular}

DISCUSSION OF VOCABULARIES

For English speaking children of 28 months three vocabularies have previously been published. The data are shown in the next table.

TABLE XIII

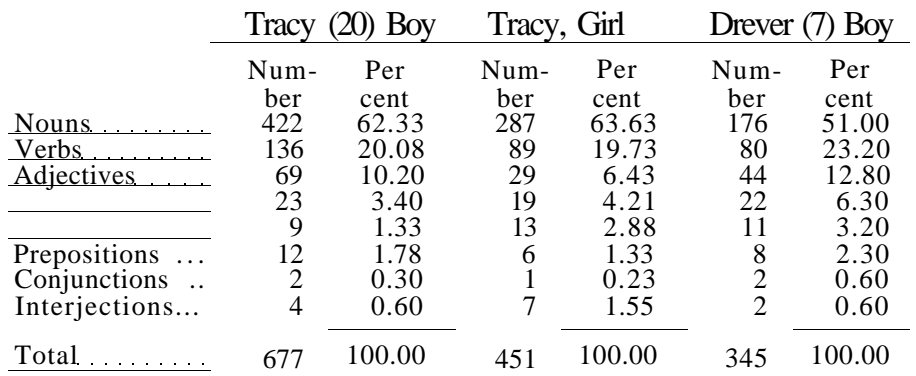


Number of Words. The average of the three lists is 491 . Since this contains proper nouns (Tracy and Drever) and inflected forms (Tracy) it is to be compared with Jane's maximum of 488, Anne's of 779 and Daphne's of 317. The average number of words at two years (20 vocabularies) is 498; at three years ( 9 vocabularies) 1,515. The average number of words in the six vocabularies at 28 months is 469 . Some reasons for the variations in the number of words used by the subjects of this paper will be discussed later.

The vocabularies cited above and the three given in this paper are those of English speaking children of whom five are American. It is interesting to compare them with the vocabulary of a German boy (19) of the same age which amounted to 310-320 words distributed as follows: Nouns $61.5 \%$, verbs $15 \%$, adjectives $8 \%$, adverbs $5.5 \%$, other parts of speech including articles and numerals $10 \%$. The list includes proper nouns.

Table XIV gives the averages for the six English speaking children.

TABLE XIV

$\begin{array}{lccccccccc} & \text { Nouns } & \text { Verbs } & \text { Adjs. } & \text { Advs. } & \text { Prns. } & \text { Prps. } & \text { Cnjs. Intjs. Total } \\ \text { Number.. } & 269 & 100 & 46 & 28 & 13 & 7 & 1 & 5 & 469 \\ \text { Percent.. } & 57.37 & 21.47 & 9.43 & 6.00 & 2.82 & 1.43 & 0.24 & 1.22 & 100.00\end{array}$

The four girls average 448 words and the two boys 511 .

\section{PARTS OF SPEECH}

Nouns and Verbs. Tracy (20) and Pelsma (17) have shown that the average per cent, of nouns in children's speech up to four years of age is about 60 and of verbs about 20 . Jane and Anne therefore use less of the former and more of the latter than most children; indeed Anne's excess of verbs is rather unusual. Daphne's deviations from the average are in the opposite direction since her percentage of nouns is above and that of the verbs below. All three children use a greater proportion of verbs than are to be found in the dictionary and probably greater than are in use by adults. The German boy used less verbs and more nouns than the American and English children, but this appears not true in general of German children.

Anne and Jane at this age readily formed present participles from verbs as fast as the latter were learned. Such forms were even made spontaneously from nouns, such as "pianoing." In Daphne's case it was impossible to tell whether she could do this or not, for since she rarely enunciated the last syllable of her words the 'ing' sound was never heard. Neither did she use the past tense; all her talking was done 
in the present form although it was clear that she knew she was describing past events. The other two children did use many past tense forms, especially Anne, but they had considerable difficulty with irregular forms so that' seed,' ' felled,' ' falled,' ' runned,' etc., were common in their speech.

A point of special interest to be noted is the comparative lack in all three vocabularies of the auxiliary verbs. In Daphne's list certain forms of the copula are also wanting.

Adjectives. Jane made a larger use of adjectives than her sister or cousin. The two former began the use of this part of speech in the twelfth month with 'good' and 'dear' respectively. These were used more imitatively than otherwise, and the word ' hot' really begins the list of adjectives in the thirteenth month. A month later this same word was the first adjective used by Daphne. Even at twenty-eight months, however, she did not use the opposite 'cold' which had in the meantime come into use by her cousins. The delicate tissues of children are peculiarly sensitive to heat so that it is not surprising to find that 'hot' was one of the most used words being even over-worked as judged by the parents. Later 'big' was much favored by all three children. Few words referring specifically to sensations of taste, hearing, organic feelings and equilibrium and few connoting moral or abstract ideas are to be found in the lists. In all these respects these children are like the majority of their kind.

Adverbs. All three children show about the same percentage of adverbs in their repertoire of words, but Jane made most use of them. It is frequently stated that this part of speech appears after the nouns, verbs and adjectives (O'Shea, 16) which was true with Jane and Daphne but not with Anne, with whom adverbial function preceded adjectival. Pelsma (17) notes another exception, as does Moore (14).

Those adverbs denoting place appear first for while 'up' and ' down' are first used of the adverbs they appear to refer in the child's mind to the place rather than to the action. Furthermore ' here' appears in use quite early. The most used of all the adverbs at this age are the modal forms and especially the negative, which were used before their complementary affirmative forms. This fact has been noted by a number of observers. Negation, indeed, is expressed preverbally at a very early age by turning away the head and by other expressive gestures. Jane was well into her third year before she used ' yes ' at all and even after that she avoided it.

Pronouns. In this class Anne has the longest list to her credit and made the most use of it. In the development of 
the idea of self each child used its given name in referring to itself, a habit which still persists with all of them. The first of the personal pronouns was ' $m y^{\prime}$ in each case but at no time did it take the place of the name or of 'me,' as instanced by O'Shea (16) in such examples as " my feel bad," and "take my to bed." The author has never heard such use of ' $m y$ ' by children although ' $m e^{\prime}$ is frequently used in such places. The egoistic ' $\mathrm{I}$ ' was first used by Jane in the twentieth month replacing the name only slowly and never quite driving it out of use. Since she first spoke of herself as Jane in the sixteenth month an interval of four months intervened between the stages of this progress. Daphne followed the same course, the time lapsing between the name and the first person singular being eight months, namely from the eighteenth to the twenty-sixth. Quite, different was Anne's procedure for she began the use of both name and pronoun in the sixteenth month. This is due without much doubt to the example of her older sister. Stern (19) instances a number of cases where first-born children began to use the name three to eighteen months before the first personal pronoun, while younger children either used the pronoun first or else both this and the name came into use simultaneously. The only English speaking child in the list is Major's boy (12) who referred to himself by name in the twenty-third month and used ' I' first in the twenty-fourth. After being once learned the aggressive pronouns ' I,' ' me,' ' my ' and 'mine ' were much used for a time by the three subjects of this paper.

Jane continued progress by taking on 'you' and 'your,' then ' us ' and ' we ' followed by ' him ' and ' she.' Anne acquired the third person singular first, then 'you' and 'we.' ' They' was later in both cases. Daphne referred to other people by name but began the use of ' our ' during a visit with her cousins. Anne's more rapid advance in this respect is again to be attributed to the influence of living with an older child.

Conjunctions. Only Anne used conjunctions. Constructions requiring the use of these are the most difficult for children to learn. Of twenty vocabularies of two-year-old children this part of speech is recorded as lacking in eight and probably is so in others; another child at 30 months is in the same category, while one of three years used only ' and.' Both Jane and Daphne used pauses to take the place of ' and' or much repetition to describe concerted action, etc. Jane began the use of the conjunction at 30 months.

Sentence Form. From what precedes much may be gathered on this topic. All the subjects showed considerable feel- 
ing for sentence structure and frequently attempted more than they could carry out with the materials at hand. An appreciable part of their talking was cast in interjectional mold and their resources were much supplemented by gesture, expression and pantomine in which again they do not differ from children in general. At times they did not take the trouble to use such small things as articles, the copula or prepositions, succeeding pretty well in being understood without them. The present time was most in their consciousness, the past was known but the future infrequently referred to. In all ways Daphne effected the most economy and simplicity of expression while Anne was the most prodigal.

Words in Common. Only 182 words are found in all three vocabularies, this comprising about 45 per cent, of Jane's list, 29 per cent, of Anne's and 60 per cent, of Daphne's. This list (printed in italics in the vocabularies) must be close to the minimum means of expression for an active child of this age. The two sisters have a larger proportion of words in common but even here individual preferences assert themselves.

Number Vocabulary. Conceptions of numbers above two were not understood by any of the children which appears to be in general the case. "Lots " meant quantity to Jane and Daphne while Anne used bravely but incorrectly many numbers in imitation of her older sister.

Color Vocabulary. In this category ' pink' and 'white' are found in Jane's list; ' black,' ' blue,' ' orange,' ' pink' and 'white ' in Anne's and 'blue' in Daphne's. The latter used the first color term at 24 months; Anne's first attempt was ' pink' in the twenty-first month and Jane also began with ' pink' in the twenty-second month, the stimulus to the new interest being in each case a new dress. Children show such variability in the ease with which they use color terms (Bateman, 4) that the differences shown by these three is not surprising. Jane and Anne agree with other children in first using pink or red while such use of blue is rather unusual. It should be stated that while pink and blue objects were correctly named, the term itself was often applied wrongly to warm shades of yellow, orange, lavender and violet in the one case and to blue-green, violet and purple in the other. This confusion did not last long, however. In contrast to these children may be cited Miss Shinn's niece (18) who readily named all the spectrum colors at 22 months. Stern's son at 28 used only schwarz and weiss and did not use the other color terms until before the third birthday. Drever's son used blue, green, red and white. 
Source of Materials. The results of an analysis of the nouns used by the children is shown in Table XV, in which the various rubrics sufficiently explain themselves.

TABLE XV

\begin{tabular}{|c|c|c|c|c|c|c|}
\hline \multirow[b]{3}{*}{ Body } & \multicolumn{3}{|c|}{ Number } & \multicolumn{3}{|c|}{ Per cent } \\
\hline & Jane & Anne & Daphne & Jane & Anne & :Daphne \\
\hline & 17 & 26 & 23 & 8.29 & 7.77 & 11.80 \\
\hline Health & 2 & 1 & 1 & .97 & .29 & .51 \\
\hline & 19 & 31 & 19 & 9.26 & 9.27 & 9.74 \\
\hline Toilet & 13 & 13 & 10 & 6.34 & 3.90 & 5.13 \\
\hline Food. & 38 & 48 & 35 & 18.52 & 14.34 & 17.95 \\
\hline Eating & 13 & 16 & 11 & 6.34 & 4.78 & 5.63 \\
\hline Play. & 23 & 37 & 18 & 11.21 & 11.05 & 9.23 \\
\hline Experience. & 2 & 6 & 3 & .97 & 1.70 & 1.53 \\
\hline I. Personal & 127 & 178 & 120 & 61.95 & 53.10 & 61.52 \\
\hline & 38 & 68 & 39 & 18.52 & 20.39 & 20.00 \\
\hline Others' things. & 3 & 6 & 7 & .46 & 1.70 & 3.59 \\
\hline II. Indoor. & 41 & 74 & 46 & 20.00 & 22.09 & 23.59 \\
\hline & 3 & 7 & 1 & 1.46 & 2.00 & .51 \\
\hline Plants. & 6 & 5 & 2 & 2.92 & 1.50 & 1.03 \\
\hline & 5 & 4 & 3 & 2.43 & 1.20 & 1.53 \\
\hline & 8 & 14 & 7 & 3.89 & 4.17 & 3.59 \\
\hline Miscellaneous & 3 & 9 & 4 & 1.46 & 2.70 & 2.05 \\
\hline III. Outdoor. . . . . & 25 & 39 & 17 & 12.20 & 11.67 & 8.72 \\
\hline IV. Books. ...... & 0 & 7 & 0 & 0.00 & 2.09 & 0.00 \\
\hline V. People . . . . . . & 5 & 13 & 7 & 2.44 & 3.89 & 3.59 \\
\hline VI. Abstract. ..... & 7 & 24 & 5 & 3.41 & 7.13 & 2.58 \\
\hline Total & 205 & 335 & 195 & 100.00 & 100.00 & 100.00 \\
\hline
\end{tabular}

The three analyses give quite dissimilar pictures. As is natural, considering the circumscribed activity of such small children, most of the material comes directly from personal experience. In this respect Jane and her cousin are about equal, both leading much the same life as only children in similar homes. Anne, on the contrary, draws much less in proportion from this source. In subheads under the general rubric Daphne was better acquainted with names of parts of the body but drew less on play. Jane had a larger percentage of words for food and eating although this did not reflect any personal excess of interest in such things. It is interesting to note the small showing of words relating to health, a condition faithfully reflecting the facts in the case. 
The next most fertile field for materials was in the house and from here Jane drew least and Daphne most, while just the reverse was true when outdoor words are taken into account. When books, people and especially abstract terms are in question, it is seen where Anne made up for neglecting what only directly concerned herself for she leads in these divisions.

Environment. In the main all three children show by the analysis the molding of each by the particular environment, this being tempered by individual reactions to the same. The greatest factor affecting Anne and not the others is that she was directly and constantly stimulated by contact with another and older child. This accounts mostly for the larger size of the vocabulary, and for some of the differences just mentioned. Anne wished to do whatever her sister did and picked up early in this way many abstract ideas and words as soon as used by Jane who was almost two years older. The same companionship kept her from being so self-centered as the other two more lonely children. Daphne in particular had had but little association with other children and" was thus thrown upon her own resources. At first thought since she lived in California one would expect her to know better the out-of-door world, but when it is known that she lived in the city and mostly within the confines of her own city lot while her small relatives had the advantage of the open fields and meadows right at hand the differences in the analysis are not surprising. Furthermore, when outdoors she was usually alone and little attention was directed to telling her the names of plants and insects while the contrary was true when in the house.

The abstract terms used were simple and with Jane and Daphne referred chiefly to quantity.

It is seen then in the main how faithfully each child reflected its own environment. The three of about the same natural ability are shown to differ in the results attained because of different circumstances surrounding them. Still other facts in this connection are brought out in what follows.

Interesting analyses of children's vocabularies have been made by Boyd (6) and Nice (15). The above table closely follows theirs, with which it may be compared.

\section{METHODS}

One of the most interesting things to follow in speech development is the spontaneous efforts of the child to acquire self-expression. Conscious imitation is the first of these to appear. Jane and her sister reached this stage during the 
seventh month, but while with Daphne the date is rather uncertain it was not much later than this. All three children developed into little chatter-boxes, repeating or trying to repeat day after day much that they heard spoken by others. Anne especially mimicked her sister, her desire to emulate in this direction being as strong as in other lines of activity. Of course the words were used purely in an imitative sense in each case for several months so that real use of language was preceded by a fairly long practice period. It was noted that during this time the principal work done was that of eliminating the many sounds which all children make early during the preverbal stage but which are of little use in speaking English. A number of observers have noted the richness and varied quality of the vowel sounds uttered by babies during the babbling period. Their vocal cords can negotiate the nasal sounds of the French and the umlauts of the German with an ease the adult can well envy. Most of this material the child must cull out in order to concentrate on the purely English vocables. However, Jane was well into the third year before she lost a certain French quality which colored her speech (Whipple, 21) while Anne was even older before the German tinge to her enunciation faded out (3). The conscious imitation or "parrot-talk" noted above seems to be characteristic of practically all the children whose language development has been traced; but Drever (7) notes an interesting exception where a child beginning to talk at sixteen months undertook the practice period after rather then before.

The author's two children as a side line from their babbling and chattering were especially fond of stringing together long series of sounds having a common vowel root. The elder girl preferred the 'og' sound of ' dog' while the younger child used ' ug' as in 'zug.' By the half hour together they would lie quietly gurgling in the one case, "oggle-woggledoggle-noggle " and in the other, " guggle-wuggle-muggle." Their cousin did not develop this kind of amusement which has been noted by others (Pelsma, 17).

A little later all the children would willingly repeat words after their parents, striving to improve on their shortcomings in enunciation. In this Jane had most success while Anne was most eager.

All three retained the use of gesture from the preverbal period as an aid to their inadequate vocabularies and this was especially true of Daphne who at 28 months was the most expressive and vivid in pantomine.

As already described, imitation and repetition were badly neglected by the three subjects during certain stages of devel- 
opment while the two younger failed to practice as well at these times. But while the two sisters recovered entirely from this depression, becoming even more energetic than before, Daphne, although proceeding to increase her linguistic resources, failed to take up imitation or practice again. Compared to her cousins she was a silent child. She did not, as they, talk and croon almost incessantly while at play. Only rarely would she repeat words or phrases after her elders while the others did so with pleasure. Neither did she have games in which words had a part or invent any new terms or find humor in sounds. At the time the three children were together (Jane aged 53 months, Anne 33 and Daphne 28) this comparative silence was quite noticeable. While, the two were noisy at their play, laughing and shouting, Daphne took her pleasure seriously although always ready to smile. Unless a new word had some immediate use, usually of close personal value, she was quite indifferent to learning it while on the contrary the other children picked up all sorts of chance words from parents or strangers. Almost invariably any word uttered in their hearing with unusual emphasis was taken up quickly. Naturally enough Daphne's neglect of practice in linguistics led to comparative lack of attainment in speech, this being especially noticeable in enunciation.

The author has already noted in another place (1-2) the use of generalization by his children between two and three years of age. Their cousin had exactly the same trait. Like them she called every kind of animal a dog and was only beginning to differentiate at 28 months. Insects were called bees and usually bad ones in reminiscence of a sting by one. Pin did for a number of diverse objects and boy and girl for still other groups. This would seem to be characteristic of the speech of little children.

A decided factor in Daphne's development was the attitude taken by her parents. They did not use much 'babytalk ' considering it foolish and a draw-back to the child but they did unconsciously without giving the matter any real thought, talk down to the baby's limited mental experience. They simplified grammar, avoided polysyllabic words and used circumlocutions. Whenever their daughter insisted (which was often) on using simple phrases or even phrases of her own they followed her lead. Thus they said " Daphne no go," " no spill soup," " want go bye-bye," etc. Some conversation included baby-talk particularly the doubling of a word to make it more emphatic or even perhaps just for the sound such as "tum-tum" (stomach), "swim-swim" and so on. The family adopted quaint babyisms and so always 
spoke of the kodak as "kolak," of bottom as "belem," of potatoes as "tates." During several enforced separations from her parents the child heard considerable baby jargon" did urns want iskle tates for ickle tum-tum?"

Naturally this kind of environment materially influenced Daphne's progress, slowing it considerably. The parents soon perceived the drawback and changed their tactics but were now met with a certain persistence on the part of the child to use the old forms which only slowly broke down with training. There is little or no value in the method of simplification. Since the child learns largely by imitation it may as well follow correct models while it is about the matter. It leaves out what it is not mentally able to use but the framework it does acquire is ready for later additions when the child's rise in ability makes such possible.

\section{ENUNCIATION}

Jane. Even from the beginning of talking Jane has spoken with a clearness rather unusual for a child. She took her time about articulation, did not hurry while she talked and so perhaps gained greater ease in enunciation. Always an observant child, her method was to calmly listen and then repeat with distinct utterance. In her baby speech very few of the inversions, substitutions and mutilations so commonly noticed were to be found. Initial ' r,' ' 1 ,' ' $\mathrm{s}$,' ' ch,' ' th ' and other ordinarily difficult sounds gave her no trouble. The principle type of pitfall was to be found in a consonant or combination of two consonants between two vowels. Thus 'pk' in napkin was vexatious as well as the ' $\mathrm{v}$ ' in shovel. But during the time considered in this paper these were the only marked flaws in enunciation. Handkerchief she never tried to say but spontaneously fastened upon " hankie" as a good substitute.

Anne. The younger child did not speak so plainly as her sister. She took less trouble usually to enunciate well although at times devoting much time to practice. The same word would be repeated over and over again in an evident persistent attempt to get it right. Anne would willingly repeat corrections to her faulty diction but usually tried to make her efforts funny so that each repetition became less like the normal and so ridiculous that the lessons ended in laughter. Her greatest fault consisted in clipping most words so that the final sound was not heard distinctly. This was especially the case with nasal sounds, with ' $y$ ' and 'ing ' but sometimes happened with vowels. ' $\mathrm{R}$ ' and ' 1 ,' however, were well enunciated. The double " $11 "$ in pillow and jelly was converted 
into ' $\mathrm{dd}$ ' or ' tt' giving ' jeddy ' or ' jetty.' Final 'ing' was often changed into 'ie' though with considerable caprice. Mother had a history of several changes, being first ' muddie,' then in succession ' mudder,' ' munner' and 'muzzer.' In the last stage, however, it was plain that the child was playing with the variations in this word and others and it often happened that four or five such variants would be used in a few minutes. Initial' $d$ ' was changed sometimes to ' $\mathrm{j}$ ' but initials ' r,' ' s,' ' th ' and ' sh ' gave practically no trouble. As already noted, many words were pronounced so as to make the vowel sounds much like the German umlauts. Anne readily noted her defects as listed above and strove to correct them thus improving greatly during the few months after the second birthday.

Daphne. In this field of linguistics Daphne had a system all her own. In the first place she had a decided preference for monosyllables, since in her vocabulary two-thirds of the words are in this class while those pronounced as one syllable in addition increase the percentage to 75 . Practically no word was spoken as having more than two sounds. Thus automobile was ' aubi,' macaroni ' maco' and canteloupe 'calo.' Of Jane's vocabulary less than half consisted of words of one syllable but of Anne's about two-thirds again so that both used about 200 polysyllables as contrasted to Daphne's 75. This preference for short words is not entirely due to the fact that they are easier for the child to say but partly because a large number of words in every day use are fortunately short. This is especially true of verbs, pronouns, prepositions and conjunctions. If the child wishes to use a word its length or difficulty of enunciation is no deterrent. Thus Daphne attempts canteloupe, automobile, rocking-chair and swimming-pool, while in Jane's list are found macaroni, mosquito, umbrella and others, and in Anne's such words as elephant, remember, sandwich, screwdriver and wheelbarrow. The use the child can make of a word or the interest it takes in the object or action connoted will prompt it to acquire difficult words.

Another characteristic of Daphne's speech was the greater use she made of ' 1 ' as compared with other children. This letter as initial was prefixed to practically all words beginning with a vowel sound. Thus she spontaneously devised for herself a sort of French definite article thus: 'l'app' (apple), ' l'eye,' ' l'ear,' ' l'e' (egg), etc. The same letter replaced initial ' $r$ ' and also initial ' $w$ ' so that ring became ' li,' rug ' lu,' rompers ' lomp ' while wipe became ' li' and wet 'le.' The same useful initial served also for the $\mathrm{Y}$ in yes. There were exceptions, however. All, ah-ha, all the words beginning with 
I, out-doors, out, watch, water, want, where and we were given the correct initial sounds.

The aspirate was rarely sounded-as initial it was replaced by $\mathrm{s}$ as in 'souse' (house), 'sorse' (horse), 'sair' (hair), ' sa' (hat). Hot had the ' 1 ' slipped in as well and became ' slaw.' Since many words were clipped of their final sound it was impossible to distinguish except by context between hand, handle, sand and sandal which were all pronounced alike. Exceptions to this were hankie and here. Initial ' th' was sometimes ' $s$ ' (thread, thumb) and sometimes ' $d$ ' (there). In ' $s h$ ' the ' $h$ ' was left out.

The initial in certain words beginning with ' $\mathrm{f}$ ' was also replaced by ' $\mathrm{s}$ ' (fig, fix, fish-man). ' $\mathrm{T}$ ' replaced initial ' $\mathrm{k}$ ' except in kodak, the ' $\mathrm{q}$ ' of quiet, even the ' $\mathrm{c}$ ' of cup and coffee. ' G' was converted into ' $d$ ' in girl and get. ' $R$ ' was another sound rarely used since it was replaced usually as initial by ' 1' and lost in broken, bread, brush, drink, drawer, etc. As final it gave less trouble but shared the common fate of such sounds in usually being left off.

In spite of the easily used ' 1 ' it was left out in blue, block, flower and floor; while the ' $\mathrm{cl}^{\prime}$ of clothes, clothes-pin and clean was changed into ' t.'

It will be noted that the child while considerably changing the accepted pronunciation of English was rather capricious about it. She did not eschew all difficult sounds nor always treat the same sound alike. It was discovered, indeed, that she used her own diction to a certain extent because she so wished and not necessarily because she was forced to adopt wrong sounds through inability to use the right. During the few weeks preceding and during the taking of the vocabulary her parents endeavored to improve her enunciation but she did not respond willingly to their efforts. As already noted she would not imitate and cared little about practice. The word ' bib' she always pronounced ' nib' and when corrected sometimes said, "No say bib, say nib." And this she calmly did. Other words could be used conventionally, too, only she preferred her own forms. As an experiment her uncle tried for a month to get her to say potatoes instead of " tates," trying all methods of cajolery, coaxing and commanding. Finally, just once, she said, "potatoes," very distinctly and then with a roguish glance followed it with, "want more tates."

This adherence to her own system had one disadvantage. She was frequently not understood. Sometimes when she volubly told a long tale with much expressive intonation even her mother could not always follow. It was evident that this 
had a discouraging effect and accounted for some of her comparative silence. Later her parents pretended not to understand certain imperfect words which they knew could be said correctly but their child understood the game and rarely gave in. Under those same circumstances her cousins would probably have acted quite differently. If it were a question of getting candy by correctly pronouncing the word, Jane would gracefully do so at once and greatly enjoy the reward; Anne would pout and hold back a little and then say the word quickly; Daphne would say nothing and do without the candy.

Such trials had an educational effect, nevertheless, for it was noted that the most recent acquisitions to Daphne's vocabulary were more correctly and distinctly pronounced. Thus sardine, teddy-bear, suit-case, lady-bug, shaving-brush and others were plainly enunciated.

\section{CONCLUSION}

In this brief survey of speech activity the author has tried to show how decidedly the language development of these three children who have much the same heredity, who in general are on a par physically and mentally, who are of the same sex and age, has been influenced by individual differences of temperament and by different factors of environment.

\section{REFERENCES}

1. Bateman, W. G. A Child's Progress in Speech. Journ. Ed. Psychol., V, 307-320.

2. Two Children's Progress in Speech. Journ. Ed. Psychol., VI, 475-493.

3. Two Children's Progress in Speech. II. Journ. Ed, Psychol., VII.

4.- The Naming of Colors by Children. Fed. Sent., XXII, 469-486.

5. BOHN, W. F. First Steps in Verbal Expression. Ped. Sent XXI 578-593.

6. BOYD, W. The Development of a Child's Vocabulary. Ped Sent XXI, 95-124.

7. DREVER, J. A Study of Children's Vocabularies. Journ. Exber Ped., HI, 34-43; 96-102.

8. GALE, H. The Vocabularies of Three Children in One Family at Two and Three Years of Age. Ped. Sent., IX, 422-455.

9. GRANT, J. R. A Child's Vocabulary and Its Growth. Ped. Sent.. XXII, 183-203.

10. HALL, W. S. First Five Hundred Days of a Child's Life. Child Study Mo., II, 585-608.

11. HOLDEN, E. S. On the Vocabularies of Children Under Two Years Trans. Am. Phil. Assn.. VIII, 58-68.

12. MAJOR. First Steps in Mental Growth. New York, 1906.

13. MICKENS, C. W. Vocabulary. Child Study Mo., Ill, 203-205.

14. MOORE, K. The Mental Development of a Child. Psych Rev Mon. Sup., No. 3, 1886. 
15. NICE, M. M. The Development of a Child's Vocabulary in Relation to Environment. Ped. Sem., XXII, 35-64.

16. O'SHEA, M. V. Linguistic Development and Education. New York, 1907.

17. PELSMA, J. R. A Child's Vocabulary and Its Development. Ped. Sent., XVII, 328-369.

18. SHINN, M. Biography of a Baby. New York, 1900.

19. STERN, C. and W. Die Kindersprache. Leipzig, 1907.

20. TRACY, F. The Psychology of Childhood. Boston, 1895.

21. WhIPPLE, G. M. The Vocabulary of a Three-year-old Boy. Ped. Sem., XVI, 1-22. 